\title{
Vehicle Routing Problem for Multiple Product Types, Compartments, and Trips with Soft Time Windows
}

\author{
P. Kabcome ${ }^{1}$ and T. Mouktonglang ${ }^{1,2}$ \\ ${ }^{1}$ Department of Mathematics, Faculty of Science, Chiang Mai University, Chiang Mai 50200, Thailand \\ ${ }^{2}$ Centre of Excellence in Mathematics, CHE, Si Ayutthaya Road, Bangkok 10400, Thailand \\ Correspondence should be addressed to T. Mouktonglang; mouktonglang.thanasak@gmail.com
}

Received 15 April 2015; Accepted 15 June 2015

Academic Editor: Chin-Chia Wu

Copyright (c) 2015 P. Kabcome and T. Mouktonglang. This is an open access article distributed under the Creative Commons Attribution License, which permits unrestricted use, distribution, and reproduction in any medium, provided the original work is properly cited.

\begin{abstract}
This paper presents a mathematical model to solve the vehicle routing problem with soft time windows (VRPSTW) and distribution of products with multiple categories. In addition, we include multiple compartments and trips. Each compartment is dedicated to a single type of product. Each vehicle is allowed to have more than one trip, as long as it corresponds to the maximum distance allowed in a workday. Numerical results show the effectiveness of our model.
\end{abstract}

\section{Introduction}

The vehicle routing problem (VRP) is a well-known problem in the operation research and combinatorial optimization presented by Dantzig and Ramser [1]. The VRP is also an important problem in the fields of transportation, distribution, and logistics. The context is planning the route to deliver goods from a central depot to customers who have placed orders for such goods. The objective of the VRP is to minimize the total route cost. According to the importance of the VRP, there are so many literatures which refer to the models and the solution of the VRP. Many papers present the different views of the problem, the different features of the system, and the different approaches to solve the problem (see [2-6] and references therein).

The vehicle routing problem with time windows (VRPTW) is a variant of the VRP (Figure 1). A solution of the VRPTW is a set of routes consisting of sequences of customers. Each route is assigned to arrive within their time windows. In addition, if a vehicle arrives before (or after) the lower (or upper) bound of the customer's time window, the vehicle cannot deliver goods to the customer (see [7-12]). An exception of VRPTW problem is that the time windows can be violated if the penalty is paid. In this case, it is called the vehicle routing problem with soft time windows (VRPSTW). In VRPSTW, each customer $i$ has a desirable time window with the highest satisfaction as $\left[a_{i}, b_{i}\right]$ and a hard time window $\left[\mathrm{LB}_{i}, \mathrm{UB}_{i}\right]$. A soft time window is shown in Figure 2. In the intervals $\left[\mathrm{LB}_{i}, a_{i}\right)$ and $\left(b_{i}, \mathrm{UB}_{i}\right]$ the service is allowed for some penalty fee. The interested reader may find more information in [13-16].

The vehicle routing problem with multiple trips (VRPMT) is another variant of the classical VRP. Each vehicle can be scheduled for more than one trip, as long as it corresponds to the maximum distance allowed in the workday [17-20].

The vehicle routing problem with multiple compartments (VRPMC) is also a special case of the VRP. Each compartment of the vehicle has a limit and is dedicated to a single type of products [21,22].

Many papers present the different views of VRP; there has not been any VRP mathematical model for multiple compartments, trips, and time windows. Several papers presented VRP for single product type. However, in [23], the author presents a model on distributing multiple items for customer. 
TABLE 1: Comparison of the problems.

\begin{tabular}{|c|c|c|c|c|c|}
\hline & VRP & VRPMC & VRPMT & VRPSTW & This study \\
\hline Vehicle & Heter/homo & Single & Heter/homo & Heter/homo & Heter/homo \\
\hline Compartment & Single & Multiple & Single & Single & Multiple \\
\hline Trip & Single & Single & Multiple & Single & Multiple \\
\hline Time window & Not considered & Not considered & Not considered & Considered & Considered \\
\hline
\end{tabular}

TABLE 2: Distance from a node $i$ to a node $j$.

\begin{tabular}{lcccccccccccccccc}
\hline $\mathrm{ds}_{i, j}$ & $o, d$ & 1 & 2 & 3 & 4 & 5 & 6 & 7 & 8 & 9 & 10 & 11 & 12 & 13 & 14 & 15 \\
\hline$o$ & 0 & 5 & 11 & 10 & 8 & 7 & 10 & 12 & 14 & 16 & 18 & 14 & 8 & 2 & 14 & 15 \\
1 & 5 & 0 & 6 & 8 & 8 & 8 & 6 & 8 & 10 & 12 & 14 & 18 & 20 & 24 & 8 & 6 \\
2 & 11 & 6 & 0 & 4 & 7 & 9 & 5 & 10 & 12 & 20 & 25 & 4 & 5 & 6 & 8 & 5 \\
3 & 10 & 8 & 4 & 0 & 6 & 10 & 7 & 9 & 12 & 11 & 10 & 8 & 3 & 8 & 10 & 12 \\
4 & 8 & 8 & 7 & 6 & 0 & 6 & 5 & 10 & 15 & 10 & 12 & 8 & 6 & 4 & 4 & 6 \\
5 & 7 & 8 & 9 & 10 & 6 & 0 & 5 & 12 & 8 & 9 & 10 & 8 & 5 & 12 & 11 & 9 \\
6 & 10 & 6 & 5 & 7 & 5 & 5 & 0 & 3 & 6 & 9 & 12 & 15 & 14 & 12 & 16 & 11 \\
7 & 12 & 8 & 10 & 9 & 10 & 12 & 3 & 0 & 10 & 12 & 14 & 16 & 18 & 14 & 8 & 2 \\
8 & 14 & 10 & 12 & 12 & 15 & 8 & 6 & 10 & 0 & 6 & 8 & 10 & 12 & 14 & 18 & 20 \\
9 & 16 & 12 & 20 & 11 & 10 & 9 & 9 & 12 & 6 & 0 & 6 & 6 & 8 & 8 & 10 & 12 \\
10 & 18 & 14 & 25 & 10 & 12 & 10 & 12 & 14 & 8 & 6 & 0 & 5 & 13 & 18 & 22 & 20 \\
11 & 14 & 18 & 4 & 8 & 8 & 8 & 15 & 16 & 10 & 6 & 5 & 0 & 4 & 8 & 12 & 14 \\
12 & 8 & 20 & 5 & 3 & 6 & 5 & 14 & 18 & 12 & 8 & 13 & 4 & 0 & 6 & 9 & 12 \\
13 & 2 & 24 & 6 & 8 & 4 & 12 & 12 & 14 & 14 & 8 & 18 & 8 & 6 & 0 & 1 & 2 \\
14 & 14 & 8 & 8 & 10 & 4 & 11 & 16 & 8 & 18 & 10 & 22 & 12 & 9 & 1 & 0 & 3 \\
15 & 15 & 6 & 5 & 12 & 6 & 9 & 11 & 2 & 20 & 12 & 20 & 14 & 12 & 2 & 3 & 0 \\
\hline
\end{tabular}

TABLE 3: The time windows for each node $i$.

\begin{tabular}{ccccccccccccccccc}
\hline & $o, d$ & 1 & 2 & 3 & 4 & 5 & 6 & 7 & 8 & 9 & 10 & 11 & 12 & 13 & 14 & 15 \\
\hline$a_{i}$ & 6 & 10 & 16 & 13 & 15 & 8 & 10 & 8 & 13 & 10 & 11 & 9 & 9 & 9 & 10 & 10 \\
$b_{i}$ & 20 & 11 & 20 & 16 & 17 & 9 & 12 & 10 & 14 & 17 & 14 & 11 & 12 & 10 & 15 & 17 \\
\hline
\end{tabular}

TABLE 4: The demand at the node $i$ for five customers.

\begin{tabular}{lcccccc}
\hline $\mathrm{dm}_{i, p}$ & $o, d$ & 1 & 2 & 3 & 4 & 5 \\
\hline$p_{1}$ & 0 & 100 & 200 & 200 & 300 & 50 \\
$p_{2}$ & 0 & 100 & 100 & 70 & 30 & 20 \\
\hline
\end{tabular}

TABLE 5: The demand at the node $i$ for ten customers.

\begin{tabular}{lccccccccccc}
\hline $\mathrm{dm}_{i, p}$ & $o, d$ & 1 & 2 & 3 & 4 & 5 & 6 & 7 & 8 & 9 & 10 \\
\hline$p_{1}$ & 0 & 50 & 100 & 120 & 40 & 30 & 60 & 80 & 50 & 130 & 40 \\
$p_{2}$ & 0 & 100 & 90 & 70 & 30 & 20 & 50 & 30 & 40 & 60 & 80 \\
\hline
\end{tabular}

TABLE 6: The demand at the node $i$ for fifteen customers.

\begin{tabular}{lcccccccccccccccc}
\hline $\mathrm{dm}_{i, p}$ & $o, d$ & 1 & 2 & 3 & 4 & 5 & 6 & 7 & 8 & 9 & 10 & 11 & 12 & 13 & 14 & 15 \\
\hline$p_{1}$ & 0 & 20 & 100 & 30 & 40 & 10 & 30 & 30 & 60 & 10 & 20 & 40 & 20 & 100 & 20 & 40 \\
$p_{2}$ & 0 & 80 & 60 & 70 & 30 & 20 & 40 & 40 & 10 & 20 & 70 & 20 & 50 & 10 & 50 & 30 \\
\hline
\end{tabular}

In this paper, we address the mathematical model for the VRP for multiple product types, compartments, and trips
TABLE 7: The capacity for compartment $t$ of vehicle $k$.

\begin{tabular}{lccc}
\hline$C_{k, t}$ & $t_{1}$ & $t_{2}$ & $t_{3}$ \\
\hline$v_{1}$ & 200 & 200 & 100 \\
$v_{2}$ & 300 & 300 & 200 \\
\hline
\end{tabular}

TABLE 8: The fixed cost of travelling $T C_{k}$ per k.m. and the capacity $\mathrm{Cp}_{k}$ of vehicle $k$.

\begin{tabular}{lcc}
\hline & $v_{1}$ & $v_{2}$ \\
\hline$T C_{k}$ & 10 & 6 \\
$\mathrm{Cp}_{k}$ & 500 & 800 \\
\hline
\end{tabular}

TABLE 9: The cost of loading for product $p$ by each vehicle $k$.

\begin{tabular}{lcc}
\hline$L C_{k, p}$ & $p_{1}$ & $p_{2}$ \\
\hline$v_{1}$ & 10 & 6 \\
$v_{2}$ & 4 & 8 \\
\hline
\end{tabular}

with soft time windows (VRPMPCMTSTW). The proposed VRP can be regarded as the extension to three problems which are

(i) the vehicle routing problem with multiple compartments,

(ii) the vehicle routing problem with multiple trips,

(iii) the vehicle routing problem with soft time windows.

The summary of the three mentioned problems is presented in Table 1. It is clear that our mathematical model solves the classical VRP since it is a special case for the VRP with multiple compartments, trips, and time windows. The time window cases are studied because they are more practical and the soft time window cases are further investigated due to the feasibility of the problem and, in some cases, due to the fact that it reduces the total cost.

In this paper, we present our mathematical models in three cases to see the development process of our study. In the first case, we consider the VRP for multiple product types, compartments, and trips where there is not a time window (model 1). Then we consider the case with time window (model 2) and, finally, the case with soft time window (model 3).

From the above content, model 3 generalizes all previous models mentioned in this paper. It is worth pointing out that another goal of the formulation is to try to formulate the mathematical models which are feasible and solvable by available solvers in a reasonable time. In this research, all models are solved by using AIMMS 3.13 on a personal 
TABLE 10: The solution for five customers.

\begin{tabular}{lccc}
\hline & Model 1 & Model 2 & Model 3 \\
\hline Objective value & 60346 & 61016 & 60476 \\
\hline Loading cost & 59860 & 60500 & 59860 \\
\hline Travel cost & 486 & 516 & 486 \\
\hline Penalty cost & - & - & 130 \\
\hline Vehicle 1 & $o \rightarrow 1 \rightarrow d$ & $o \rightarrow 5 \rightarrow d$ & $o \rightarrow 5 \rightarrow d$ \\
Trip 1 & $o \rightarrow 5 \rightarrow d$ & $o \rightarrow 1 \rightarrow d$ & $o \rightarrow 1 \rightarrow d$ \\
Trip 2 & $o \rightarrow 3 \rightarrow 2 \rightarrow d$ & $o \rightarrow 4 \rightarrow 3 \rightarrow d$ & $o \rightarrow 4 \rightarrow d$ \\
Vehicle 2 & $o \rightarrow 4 \rightarrow d$ & $o \rightarrow 2 \rightarrow d$ & $o \rightarrow 3 \rightarrow 2 \rightarrow d$ \\
Trip 1 & 0.37 & 0.27 & 0.5 \\
Trip 2 & & & $o$ \\
\hline Times (seconds) & & & 0 \\
\hline
\end{tabular}

TABLE 11: The solution for ten customers.

\begin{tabular}{|c|c|c|c|}
\hline & Model 1 & Model 2 & Model 3 \\
\hline Objective value & 106574 & 110744 & 109130 \\
\hline Loading cost & 105620 & 109780 & 107840 \\
\hline Travel cost & 954 & 964 & 1010 \\
\hline Penalty cost & - & - & 280 \\
\hline \multicolumn{4}{|l|}{ Vehicle 1} \\
\hline Trip 1 & $o \rightarrow 5 \rightarrow 10 \rightarrow d$ & $o \rightarrow 7 \rightarrow d$ & $o \rightarrow 6 \rightarrow 7 \rightarrow d$ \\
\hline Trip 2 & $o \rightarrow 4 \rightarrow d$ & $o \rightarrow 1 \rightarrow 6 \rightarrow 8 \rightarrow d$ & $o \rightarrow 1 \rightarrow 10 \rightarrow d$ \\
\hline \multicolumn{4}{|l|}{ Vehicle 2} \\
\hline Trip 1 & $o \rightarrow 6 \rightarrow 8 \rightarrow 9 \rightarrow d$ & $o \rightarrow 5 \rightarrow 9 \rightarrow 10 \rightarrow d$ & $o \rightarrow 5 \rightarrow 9 \rightarrow 8 \rightarrow d$ \\
\hline Trip 2 & $o \rightarrow 1 \rightarrow 2 \rightarrow 3 \rightarrow 7 \rightarrow d$ & $o \rightarrow 3 \rightarrow 2 \rightarrow 4 \rightarrow d$ & $o \rightarrow 3 \rightarrow 2 \rightarrow 4 \rightarrow d$ \\
\hline Times (seconds) & 28 & 5 & 11 \\
\hline
\end{tabular}

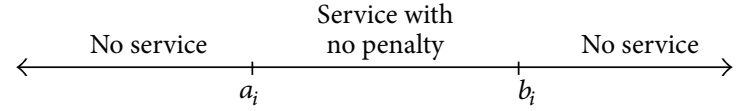

FIGURE 1: Service time interval in VRPTW.

\begin{tabular}{|c|c|c|c|c|}
\hline No service & $\begin{array}{c}\text { Service with } \\
\text { penalty }\end{array}$ & $\begin{array}{l}\text { Service with } \\
\text { no penalty }\end{array}$ & $\begin{array}{l}\text { Service with } \\
\text { penalty }\end{array}$ & No service \\
\hline & $\omega_{l}$ & & $b_{i}$ & $\mathrm{UB}_{i}$ \\
\hline
\end{tabular}

FIGURE 2: Service time interval in VRPSTW.

computer. The remainder of the paper is organized as follows. In Section 2, the problem is defined. Also the assumptions, notations, and mathematical models are presented. Section 3 contains the computational results of our formulations. We use the same set of data from [23] to examine the feasibility and solutions of the model. Finally, in Section 4, we summarize our conclusion and provide pointers for further research.

\section{Problem Formulation}

In this section, we present a mathematical model for the vehicle routing problem for multiple product types, compartments, and trips with soft time windows. The following notations are introduced to formulate the mathematical model.

Sets (Indexes). Let $G(N, A)$ be a directed graph where $N$ is a set of vertices and $A$ is a set of $\operatorname{arcs}(i, j)$ representing the connections between the depot and customers and among the customers, where $i \neq j$. Indeed, let

$N=\{o, d, 1,2,3, \ldots, n\}$ be a node set (o is origin and $d$ is destination),

$N^{*}=N \backslash\{o, d\}$,

$A(i, j): i, j \in N, i \neq j$ be the arc set,

$K=\{1,2,3, \ldots, v\}$ be a set of vehicles,

$P=\{1,2,3, \ldots, p\}$ be a set of product types,

$T=\{1,2,3 \ldots, t\}$ be a set of compartments,

$R=\{1,2,3, \ldots, r\}$ be a set of trips.

\section{Parameters. Let}

$\mathrm{ds}_{i, j}$ be a distance between customers $i$ and $j$,

$t_{i, j}$ be a time (hour) from customers $i$ to $j$ (e.g., $t_{i, j}=$ $\mathrm{ds}_{i, j} / v$ when $v$ means traveling speed of each vehicle), $\mathrm{dm}_{i, p}$ be the demand of the customer $i$ in product $p$, 
$C \mathrm{p}_{k, t}$ be the capacity of a compartment $t$ in the vehicle $k$,

$M$ be an arbitrary big number (e.g., $M=\sum_{k} \sum_{t} \mathrm{Cp}_{k, t}$ ),

$T C_{k}$ be the fixed cost per kilometer of running of each vehicle,

$L C_{k, p}$ be the cost per unit travel per unit of load of product type by the vehicle,

$s_{i}$ be a service time at the customer $i$,

$a_{i}$ be the lower bound of soft time window for the node $i$,

$b_{i}$ be the upper bound of soft time window for the node $i$,

$\mathrm{LB}_{i}$ be the lower bound of hard time window for the node $i$,

$\mathrm{UB}_{i}$ be the upper bound of hard time window for the node $i$,

$P_{l}$ be penalty cost per hour for the earliness,

$P_{u}$ be penalty cost per hour for the lateness.
Decision Variables. Consider

$$
\begin{aligned}
& X_{k, r, i, j} \\
& = \begin{cases}1, & \text { if vehicle } k \text { travels directly from node } i \text { to } j \text { in trip } r \\
0, & \text { otherwise, }\end{cases}
\end{aligned}
$$

$X T_{k, r, i, j, p}$ is the quantity of product type $p$ to be transported from node $i$ to node $j$ by vehicle $k$ in trip $r$,

$X L_{k, r, i, p}$ is the quantity of product type $p$ to be disposed at node $i$ by vehicle $k$ in trip $r$,

$W_{k, r, i}$ is the starting time of service in node $i$ by the vehicle $k$ in trip $r$,

$W l_{k, r, i}=\max \left\{a_{i}-W_{k, r, i}, 0\right\}$ is the violation degree of a lower bound of soft time window,

$W u_{k, r, i}=\max \left\{W_{k, r, i}-b_{i}, 0\right\}$ is the violation degree of an upper bound of soft time window, and

$$
U_{k, r, t, p}= \begin{cases}1, & \text { if a vehicle } k \text { delivers a product } p \text { in a compartment } t \text { in a trip } r \\ 0, & \text { otherwise. }\end{cases}
$$

The problem is solved under the following assumptions:

(1) Every customer can be served by one and only one vehicle.

(2) Every vehicle starts and ends only at the central depot.

(3) The total demand on one particular route must not exceed the capacity of the vehicle.

(4) Each customer must be served in the related hard time window.
(5) A service (wait) time in each customer is allowed.

(6) The hard time windows must not be violated.

(7) The soft time windows can be violated at a fixed cost.

Mathematical Model. The vehicle routing problem for multiple product types, compartments, and trips (model 1) can then be stated mathematically as follows:

$$
\begin{aligned}
\min & \left\{\sum_{k} \sum_{r} \sum_{(i, j) \in A} \mathrm{ds}_{i, j} X_{k, r, i, j} T C_{k}+\sum_{k} \sum_{r} \sum_{p} \sum_{(i, j) \in A} X T_{k, r, i, j, p} \mathrm{ds}_{i, j} L C_{k, p}\right\} \\
\text { subject to: } & \sum_{j} X_{k, r, i, j}-\sum_{j} X_{k, r, i, j}= \begin{cases}-1, & i=o ; \\
1, & i=d ; \quad \forall i \in N, \quad \forall k \in K, \quad \forall r \in R, \\
0, & \text { otherwise; }\end{cases} \\
& \sum_{k} \sum_{r} \sum_{j} X_{k, r, i, j}=1 ; \quad \forall i \in N^{*}, \\
& \sum_{j} X_{k, r, i, j} \leq 1 ; \quad \forall i \in N^{*}, \forall k \in K, \forall r \in R,
\end{aligned}
$$




$$
\begin{aligned}
& \sum_{j \in N^{*}} X T_{k, r, o, j, p} \leq \sum_{t} U_{k, r, t, p} \mathrm{Cp}_{k, t} ; \quad \forall k \in K, \forall r \in R, \forall p \in P, \\
& \sum_{k} X L_{k, r, i, p}=\mathrm{dm}_{i, p} ; \quad \forall i \in N^{*}, \forall p \in P, \\
& \sum_{k} \sum_{r} \sum_{j} X T_{k, r, i, j, p}=\sum_{j \in N^{*}} \mathrm{dm}_{j, p} ; \quad \forall i \in N^{*}, \forall p \in P, \\
& \sum_{i} X T_{k, r, i, n, p}-X L_{k, r, n, p}=\sum_{j} X T_{k, r, n, j, p} ; \quad \forall i \in N^{*}, \forall k \in K, \forall r \in R, \forall p \in P, \\
& X T_{k, r, i, j, p} \leq X X_{k, r, i, j} M ; \quad \forall i, j \in N, \forall k \in K, \forall r \in R, \forall p \in P, \\
& X T_{k, r, i, d, p}=0 ; \quad \forall i \in N, \forall k \in K, \forall r \in R, \forall p \in P, \\
& X_{k, i, i}=0 ; \quad \forall i \in N, \forall k \in K, \\
& X_{k, o, d}=0 ; \quad \forall k \in K, \\
& \sum_{p} U_{k, r, t, p} \leq 1 ; \quad \forall t \in T \forall k \in K .
\end{aligned}
$$

We minimize objective function (3) which consists of two components. The first component represents traveling cost. It depends on distances covered by vehicles and the fixed costs per unit distance. The second component is defined as the fleet cost which is equal to the sum of the costs related to the loading and distance. Constraints (4) are the flow conservation of a vehicle. That is, for $i=o$, every vehicle starts only once at the depot and no arc terminates at node $o$, and also, for $i=d$, every vehicle ends only once at the depot and no arc originates from node $d$, and $i \in N^{*}$ indicate that exactly one vehicle enters and leaves each customer node. Constraints (5) require the fact that each customer is serviced exactly once. Constraints (6) state that all customers must be assigned to at most one vehicle. Constraints (7) ensure that the total number of quantity transported does not exceed the vehicle's capacity. Constraints (8) state that the sum of the quantities of product type disposed at a customer by all the vehicles must be equal to the demand for product $p$. Constraints (9) represent the sum of quantities of the product type to be transported by the vehicle to the customer from the depot node $(o)$. It should in total be greater than the total sum of the demands at all customers. Constraints (10) state that the quantity of product transported to a customer minus the quantity disposed at that customer must be equal to the quantity transported to the next customer by the vehicle. Constraints (11) represent the transported quantity. That is, variable $X T_{k, i, j, p}=0$ if route segment $(i, j)$ is not used by vehicle $k$; otherwise it should not exceed the total tonnage capacity of vehicle $k$. Constraints (12) state that each vehicle must not load at the depot. Constraints (13) and (14) are the flow conservation constraints which describe the vehicle path. Constraints (15) define a limit for each compartment to be dedicated to a single type of product.

This model can be extended to the model called vehicle routing problem for multiple product types, compartments, and trips with time windows (model 2). In the next model, we include the time windows for each node. Consider

$$
\begin{aligned}
\left(W_{k, r, i}+t_{i, j}+s_{i}-W_{k, r, j}\right) & X_{k, r, i, j} \leq 0 ; \\
& \forall i, j \in N, \forall k \in K, \forall r \in R .
\end{aligned}
$$

Constraints (16) ensure feasibility of the time schedule; that is, $W_{k, r, j}$ must be greater than or equal to $W_{k, r, i}+t_{i, j}+$ $s_{i}$ whenever the vehicle $k$ travels from $i$ to $j$. By rewriting constraints (16), they can be replaced with the following constraints:

$$
\begin{aligned}
& W_{k, r, i}-W_{k, r, j}+\left(b_{i}+t_{i, j}+s_{i}-a_{j}\right) X_{k, r, i, j} \leq b_{i}-a_{j} \\
& \forall i, j \in N, \forall k \in K, \forall r \in R .
\end{aligned}
$$

$a_{i} \leq W_{k, r, i} \leq b_{i} ; \quad \forall i \in N^{*}, \forall k \in K, \forall r \in R$.

Constraints (18) ensure that the starting time of a service does not violate the given hard time window.

Proposition 1. Constraints

$$
\begin{aligned}
& W_{k, r, i}+t_{i, j}+s_{i}-W_{k, r, j} \\
& \leq\left(1-X_{k, r, i, j}\right) \max \left(0, b_{i}+t_{i, j}-a_{j}\right) ; \\
& \quad \forall i, j \in N, \forall k \in K, \forall r \in R
\end{aligned}
$$

are valid for model 2. Moreover, any $W_{k, r, i}, W_{k, r, j}, X_{k, r, i, j}$ that satisfies constraints (19) satisfies constraints (17).

Proof of Proposition 1 is straightforward and, thus, we omit it.

The vehicle routing problem for multiple product types, compartments, and trips with time windows can be stated as follows: 


$$
\min \left\{\sum_{k} \sum_{r} \sum_{(i, j) \in A} \mathrm{ds}_{i, j} X_{k, r, i, j} T C_{k}+\sum_{k} \sum_{r} \sum_{p} \sum_{(i, j) \in A} X T_{k, r, i, j, p} \mathrm{ds}_{i, j} L C_{k, p}\right\}
$$

subject to:

$$
\begin{aligned}
& (4)-(15),(18),(19) \\
& W_{k, r+1, o} \geq W_{k, r, d} ; \quad \forall k \in K .
\end{aligned}
$$

Constraints (18) assure that the starting time of services does not violate the given hard time windows. Constraints (21) assure that the starting time in next trip of each vehicle is satisfied.
Finally we consider the complete the generalization of all models mentioned in this paper. We include soft time windows for each time window allowing the vehicle to enter the depot earlier or later with some penalties. The vehicle routing problem for multiple product types, compartments, and trips with soft time windows (model 3) can then be stated mathematically as

$$
\min \left\{\sum_{k} \sum_{r} \sum_{(i, j) \in A} \mathrm{ds}_{i, j} X_{k, r, i, j} T C_{k}+\sum_{k} \sum_{r} \sum_{p} \sum_{(i, j) \in A} X T_{k, r, i, j, p} \mathrm{ds}_{i, j} L C_{k, p}+\sum_{k} \sum_{r} \sum_{i}\left(P l W l_{k, r, i}+P u W u_{k, r, i}\right)\right\}
$$

subject to: (4) $-(15),(22)$,

$$
\begin{aligned}
& W_{k, r, i}+t_{i, j}+s_{i}-W_{k, r, j} \leq\left(1-X_{k, r, i, j}\right) \max \left(0, \mathrm{UB}_{i}+t_{i, j}-\mathrm{LB}_{j}\right) ; \quad \forall i, j \in N, \forall k \in K, \forall r \in R, \\
& \mathrm{LB}_{i} \leq W_{k, r, i} \leq \mathrm{UB}_{i} ; \quad \forall i \in N^{*}, \forall k \in K, \forall r \in R, \\
& W l_{k, r, i} \geq a_{i}-W_{k, r, i} ; \quad \forall i, j \in N^{*}, \forall k \in K, \\
& W u_{k, r, i} \geq W_{k, r, i}-b_{i} ; \quad \forall i, j \in N^{*}, \forall k \in K .
\end{aligned}
$$

The objective function differs from models 1 and 2 by adding the third component. The objective function is to minimize the sum of the cost of traveled distances, total of costs related to loading products, and the total penalties of the outrage from soft time windows. We then replaced constraints (19) and (18) by constraints (23). Constraints (24) determine the violation degree of the lower and upper bound of soft time windows for each node.

\section{Numerical Results}

Let $o, 1,2,3, \ldots, 15, d$ be nodes where $o$ and $d$ are the same nodes. First of all, we consider test problems in order to observe the feasibility and solvability of the models. We test our models on the sets of 5,10 , and 15 customers. We use the same set of data from [23] to examine the feasibility and solutions of the model. Clearly, our models are more general than the one in [23]. However, we use this data set as a special case. Second of all, we randomly generate demands, service times, time windows of the customers, and distances between nodes as in Tables 2-6. The demands of customers are given in Tables 4-6.

We assume that there are two vehicle types: $v_{1}$ and $v_{2}$ and two product types: $p_{1}$ and $p_{2}$. For a general number of vehicles and products, some modifications can be easily done. We define our compartment in Table 7 . We only allow a maximum of two trips per day for each vehicle. Obviously, for more general maximum number of trips per day, a small modification can be easily done as well too. For the parameters related to cost function, we let the unit penalty of the earliness $P_{e}$ be 100 units and we let unit penalty of lateness $P_{l}$ be 200 units. The cost of travel and loading are shown in Tables 8 and 9. Let also the lower bound of hard time window for the node $i$ be $\mathrm{LB}_{i}=a_{i}-0.5$. The upper bound of hard time window for the node $i$ is $\mathrm{UB}_{i}=b_{i}+1$ and the service time for each node $s_{i}$ is 10 unit times and $v$ is 20 -unit distance per unit time.

All models have been coded and solved by AIMMS 3.13 [24]. They were run on a computer equipped with a processor Intel Core i5 at $2.3 \mathrm{GHz}$ and $2 \mathrm{~GB}$ of RAM memory. The optimal solutions are obtained in Tables 10-12. The average run time for each case is included in Tables 10-12. As one can see, our model can be executed by an available solver on a personal computer in a reasonable time. We present the detailed optimal results of model 3 in the case of 15 customers for the effectiveness of the model. The optimal solutions are presented in Tables 13-16. 
TABLE 12: The solution for fifteen customers.

\begin{tabular}{|c|c|c|c|}
\hline & Model 1 & Model 2 & Model 3 \\
\hline Objective value & 74548 & 107098 & 83228 \\
\hline Loading cost & 73540 & 105900 & 81760 \\
\hline Travel cost & 1008 & 1198 & 1078 \\
\hline Penalty cost & - & - & 390 \\
\hline \multicolumn{4}{|l|}{ Vehicle 1} \\
\hline $\begin{array}{l}\text { Trip } 1 \\
\text { Trip } 2\end{array}$ & $\begin{array}{c}o \rightarrow 4 \rightarrow 5 \rightarrow d \\
o \rightarrow 12 \rightarrow 3 \rightarrow 10 \rightarrow d\end{array}$ & $\begin{array}{c}o \rightarrow 5 \rightarrow 7 \rightarrow d \\
o \rightarrow 1 \rightarrow 6 \underset{d}{\rightarrow} \rightarrow 14 \rightarrow\end{array}$ & $\begin{array}{c}o \rightarrow 5 \rightarrow 12 \rightarrow 11 \rightarrow d \\
o \rightarrow 1 \rightarrow 10 \rightarrow d\end{array}$ \\
\hline \multicolumn{4}{|l|}{ Vehicle 2} \\
\hline Trip 1 & $o \rightarrow 1 \rightarrow 2 \underset{d}{\rightarrow} 11 \rightarrow 9 \rightarrow$ & $\begin{array}{c}o \rightarrow 13 \rightarrow 12 \rightarrow 11 \rightarrow \\
10 \rightarrow 9 \rightarrow 8 \rightarrow d\end{array}$ & $\begin{array}{l}o \rightarrow 13 \rightarrow 14 \rightarrow 15 \rightarrow \\
7 \rightarrow 6 \rightarrow 8 \rightarrow 9 \rightarrow d\end{array}$ \\
\hline Trip 2 & $\begin{array}{c}o \rightarrow 13 \rightarrow 14 \rightarrow 15 \rightarrow \\
7 \rightarrow 6 \rightarrow 8 \rightarrow d\end{array}$ & $o \rightarrow 4 \rightarrow 2 \rightarrow 15 \rightarrow d$ & $o \rightarrow 3 \rightarrow 2 \rightarrow 4 \rightarrow d$ \\
\hline Times (seconds) & 287 & 1320 & 1599 \\
\hline
\end{tabular}

TABLE 13: The service time at node $i$ by a vehicle $k$ in a trip $r$.

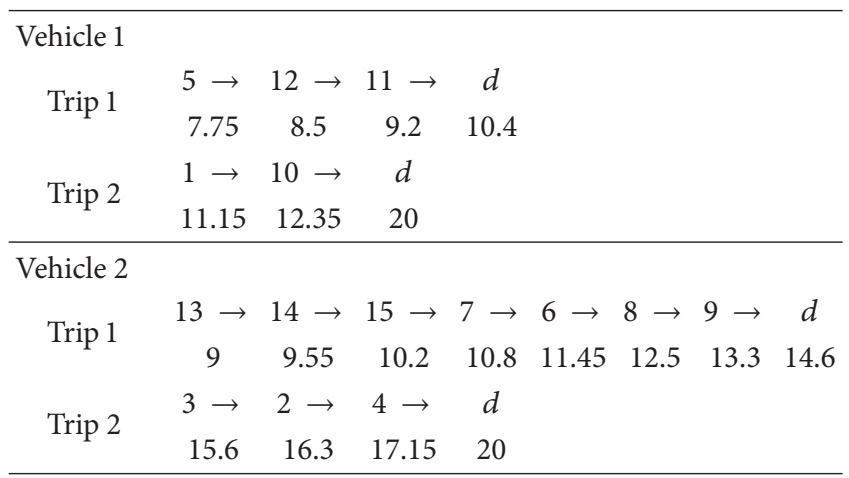

TABLE 14: The violation degree of the bound for soft time at node $i$.

\begin{tabular}{|c|c|c|c|c|c|c|c|c|c|c|c|c|}
\hline$W l_{k, r, i}$ & 1 & 23 & 4 & 5 & 6 & 7 & 8 & $\begin{array}{lll}9 & 10 & 11\end{array}$ & 12 & 13 & 14 & 15 \\
\hline \multicolumn{13}{|l|}{ Vehicle 1} \\
\hline Trip 1 & & & & 0.25 & & & & & 0.5 & & & \\
\hline \multicolumn{13}{|l|}{ Trip 2} \\
\hline \multicolumn{13}{|l|}{ Vehicle 2} \\
\hline Trip 1 & & & & & & & 0.5 & & & & 0.45 & \\
\hline \multicolumn{13}{|l|}{ Trip 2} \\
\hline$W u_{k, r, i}$ & 1 & 23 & 4 & 5 & 6 & 7 & 8 & $\begin{array}{llll}9 & 10 & 11 \\
\end{array}$ & 12 & 13 & 14 & 15 \\
\hline \multicolumn{13}{|l|}{ Vehicle 1} \\
\hline \multicolumn{13}{|l|}{ Trip 1} \\
\hline Trip 2 & 0.15 & & & & & & & & & & & \\
\hline \multicolumn{13}{|l|}{ Vehicle 2} \\
\hline Trip 1 & & & & & & 0.8 & & & & & & \\
\hline Trip 2 & & & 0.15 & & & & & & & & & \\
\hline
\end{tabular}

From the numerical examples, it is to be expected that the optimal values for the case where time windows are allowed are less than the case where the time windows are not allowed. Detailed results on each problem can be found in Tables 10,
TABLE 15: The load of the product $p$ in a compartment $t$ of vehicle $k$.

\begin{tabular}{lcc}
\hline$U_{k, r, t, p}$ & Product 1 & Product 2 \\
\hline Vehicle 1, trip 1 & 1 & \\
Compartment 1 & 1 & \\
Compartment 2 & & \\
Compartment 3 & & 1 \\
Vehicle 1, trip 2 & & 1 \\
Compartment 1 & & \\
Compartment 2 & 1 & \\
Compartment 3 & & \\
Vehicle 2, trip 1 & 1 & \\
Compartment 1 & 1 & \\
Compartment 2 & & \\
Compartment 3 & & \\
Vehicle 2, trip 2 & 1 & \\
Compartment 1 & 1 & \\
Compartment 2 & & \\
Compartment 3 & &
\end{tabular}

11 , and 12 for the of cases models 1 and 2. However, there are a few cases where the time windows do not have any effect. In these cases, the optimal values for both cases should be coincided. As for the case with soft time windows, together with the penalty, we expect that the optimal value of the soft time windows case should obviously be less than the (hard) time windows since the feasible solution set for the soft time windows is strictly larger than the feasible solution set or the time windows. The results of this experiment are shown in Tables 10, 11, and 12 in cases of models 2 and 3.

\section{Conclusion}

In this paper, we present a mathematical model for the vehicle routing problem for multiple product types, compartments, and trips with soft time windows. We have verified 
TABLE 16: The quantity of product $p$ moved from node $i$ to node $j$ and delivered at node $i$ for fifteen customers.

\begin{tabular}{|c|c|c|c|c|}
\hline \multirow[b]{2}{*}{$X T_{k, r, i, j, p}$} & \multicolumn{2}{|c|}{ Product 1} & \multicolumn{2}{|c|}{ Product 2} \\
\hline & $\begin{array}{c}\text { Moved } \\
\text { from } i \text { to } j\end{array}$ & $\begin{array}{c}\text { Delivered } \\
\text { at } j\end{array}$ & $\begin{array}{c}\text { Moved } \\
\text { from } i \text { to } j\end{array}$ & $\begin{array}{c}\text { Delivered } \\
\text { at } j\end{array}$ \\
\hline \multicolumn{5}{|c|}{ Vehicle 1, trip 1} \\
\hline$o-5$ & 70 & 10 & 90 & 20 \\
\hline $5-12$ & 60 & 20 & 70 & 50 \\
\hline $12-11$ & 40 & 40 & 20 & 20 \\
\hline \multicolumn{5}{|c|}{ Vehicle 1 , trip 2} \\
\hline$o-1$ & 40 & 20 & 150 & 80 \\
\hline $1-10$ & 20 & 20 & 70 & 70 \\
\hline \multicolumn{5}{|c|}{ Vehicle 2 , trip 1} \\
\hline$o-13$ & 290 & 100 & 200 & 10 \\
\hline $13-14$ & 190 & 20 & 190 & 50 \\
\hline $14-15$ & 170 & 40 & 140 & 30 \\
\hline $15-7$ & 130 & 30 & 110 & 40 \\
\hline $7-6$ & 100 & 30 & 70 & 40 \\
\hline $6-8$ & 70 & 60 & 30 & 10 \\
\hline $8-9$ & 10 & 10 & 20 & 20 \\
\hline \multicolumn{5}{|c|}{ Vehicle 2 , trip 2} \\
\hline$o-3$ & 170 & 30 & 160 & 70 \\
\hline $3-2$ & 140 & 100 & 90 & 60 \\
\hline $2-4$ & 40 & 40 & 30 & 30 \\
\hline
\end{tabular}

our feasibilities and attended the optimal solution for the problems. We use the software package AIMMS to solve the problems on a personal computer. The run times are acceptable. Numerical results show the effectiveness of our model.

\section{Conflict of Interests}

The authors declare that there is no conflict of interests regarding the publication of this paper.

\section{Acknowledgments}

The authors would like to thank the referees for their valuable comments and suggestions. This research is supported by the Centre of Excellence in Mathematics, the Commission on Higher Education, Thailand.

\section{References}

[1] G. B. Dantzig and J. H. Ramser, "The truck dispatching problem," Management Science, vol. 6, pp. 80-91, 1959.

[2] M. Ball, T. Maganti, C. Monma, and G. Nemhasuser, Handbook in Operations Research and Management Science, 8: Network Routing, 1995.

[3] J.-F. Cordeau, M. Gendreau, G. Laporte, J.-Y. Potvin, and F. Semet, "A guide to vehicle routing heuristics," Journal of the Operational Research Society, vol. 53, no. 5, pp. 512-522, 2002.
[4] M. Gendrau, G. Laport, and J. Potvin, "Vehicle routing: modern heuristics," in Local Search in Combinatorial Optimization, E. Aarts and J. Lenstra, Eds., pp. 311-336, Wiley, London, UK, 1997.

[5] B. Golden and A. Assad, Vehicle Routing: Methods and Studies, Elsevier Science, Amsterdam, The Netherlands, 1988.

[6] P. Toth and D. Vigo, The Vehicle Routing Problem, vol. 9 of SIAM Monographs on Discrete Mathematics and Applications, 2002.

[7] J.-F. Cordeau, G. Laporte, and A. Mercier, "A unified tabu search heuristic for vehicle routing problems with time windows," Journal of the Operational Research Society, vol. 52, no. 8, pp. 928-936, 2001.

[8] M. Desrochers, J. Desrosiers, and M. Solomon, "A new optimization algorithm for the vehicle routing problem with time windows," Operations Research, vol. 40, no. 2, pp. 342-354, 1992.

[9] J. Homberger and H. Gehring, "Two evolutionary metaheuristics for the vehicle routing problem with time windows," INFOR Journal, vol. 37, pp. 297-318, 1999.

[10] G. Ioannou, M. Kritikos, and G. Prastacos, "A greedy lookahead heuristic for the vehicle routing problem with time windows," Journal of the Operational Research Society, vol. 52, no. 5, pp. 523-537, 2001.

[11] N. Kohl and O. B. Madsen, "An optimization algorithm for the vehicle routing problem with time windows based on Lagrangian relaxation," Operations Research, vol. 45, no. 3, pp. 395-406, 1997.

[12] M. M. Solomon, "Algorithms for the vehicle routing and scheduling problems with time window constraints," Operations Research, vol. 35, no. 2, pp. 254-265, 1987.

[13] N. Balakrishnan, "Simple heuristics for the vehicle routeing problem with soft time windows," Journal of the Operational Research Society, vol. 44, no. 3, pp. 279-287, 1993.

[14] G. Ioannou, M. Kritikos, and G. Prastacos, "A problem generator-solver heuristic for vehicle routing with soft time windows," Omega, vol. 31, no. 1, pp. 41-53, 2003.

[15] H. Min, "A multiobjective vehicle routing problem with soft time windows: the case of a public library distribution system," Socio-Economic Planning Sciences, vol. 25, no. 3, pp. 179-188, 1991.

[16] É. Taillard, P. Badeau, M. Gendreau, F. Guertin, and J.-Y. Potvin, "A tabu search heuristic for the vehicle routing problem with soft time windows," Transportation Science, vol. 31, no. 2, pp. 170-186, 1997.

[17] B. Fleischmann, "The vehicle routing problem with multiple use of vehicles," Working Paper, Fachbereich Wirtschaftswissenschaften, University Hamburg, Hamburg, Germany, 1990.

[18] R. J. Petch and S. Salhi, "A multi-phase constructive heuristic for the vehicle routing problem with multiple trips," Discrete Applied Mathematics, vol. 133, no. 1-3, pp. 69-92, 2003.

[19] A. Olivera and O. Viera, "Adaptive memory programming for the vehicle routing problem with multiple trips," Computers \& Operations Research, vol. 34, no. 1, pp. 28-47, 2007.

[20] C. Prins, "Efficient heuristics for the heterogeneous fleet multitrip VRP with application to a large-scale real case," Journal of Mathematical Modelling and Algorithms, vol. 1, no. 2, pp. 135$150,2002$.

[21] A. E. Fallahi, C. Prins, and R. Wolfler Calvo, "A memetic algorithm and a tabu search for the multi-compartment vehicle routing problem," Computers \& Operations Research, vol. 35, no. 5, pp. 1725-1741, 2008.

[22] U. Derigs, J. Gottlieb, J. Kalkoff, M. Piesche, F. Rothlauf, and U. Vogel, "Vehicle routing with compartments: applications, 
modelling and heuristics," OR Spectrum, vol. 33, no. 4, pp. 885914, 2011.

[23] C. G. Tapia, "A mixed-binary linear model for fleet routing and multiple product-type distribution," Philippine Management Review, vol. 6, no. 1, pp. 75-86, 1995-1996.

[24] M. Roelofs and J. Bisschop, AIMMS Language Reference, Paragon Decision Technology, 2013. 


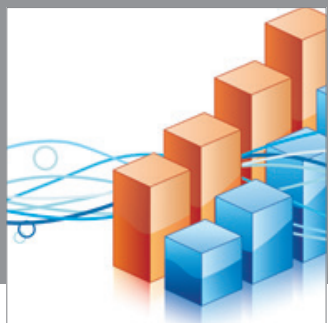

Advances in

Operations Research

mansans

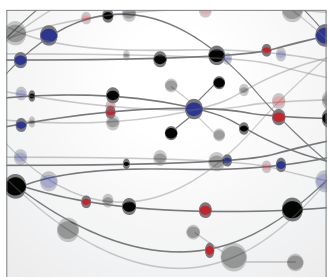

The Scientific World Journal
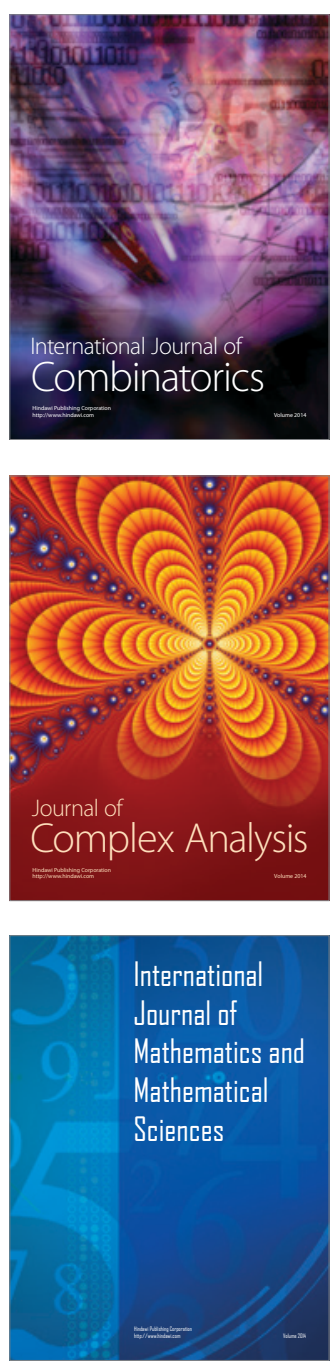
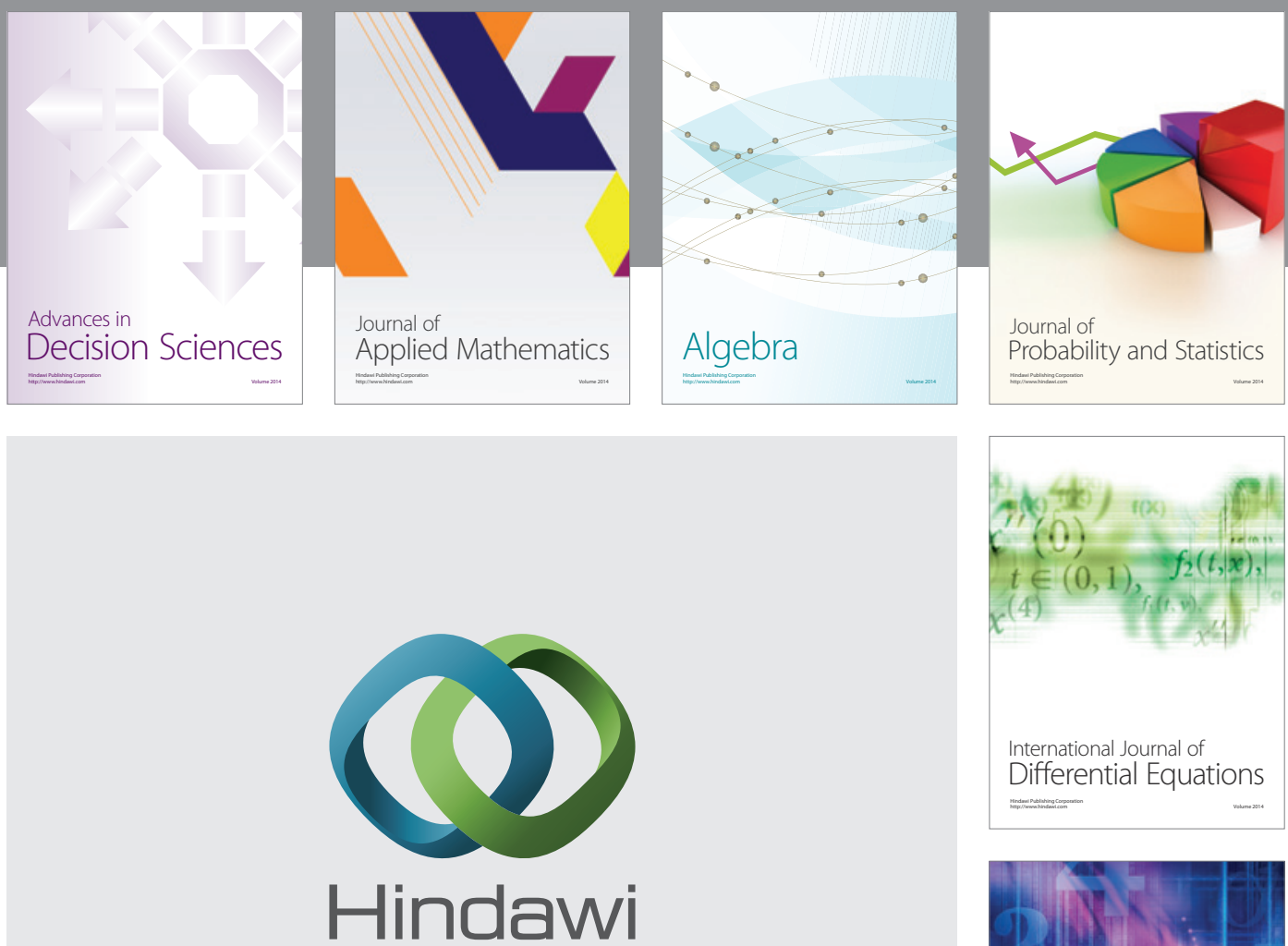

Submit your manuscripts at http://www.hindawi.com
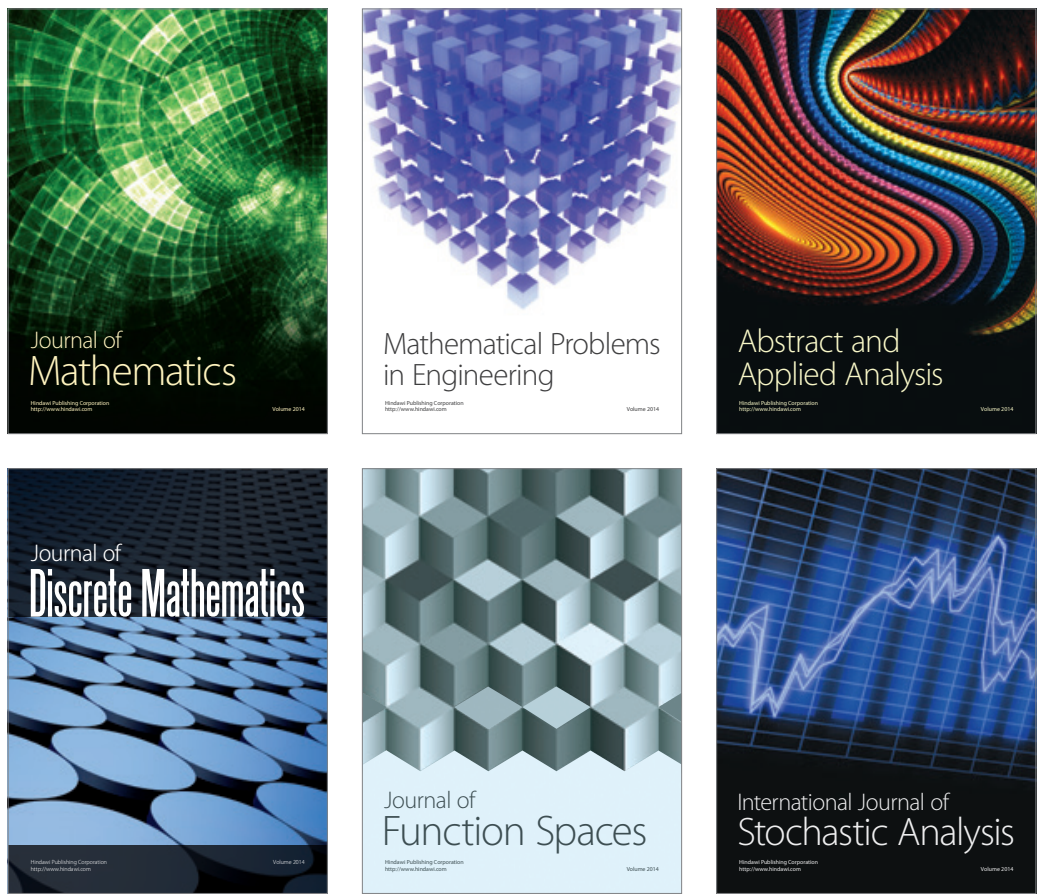

Journal of

Function Spaces

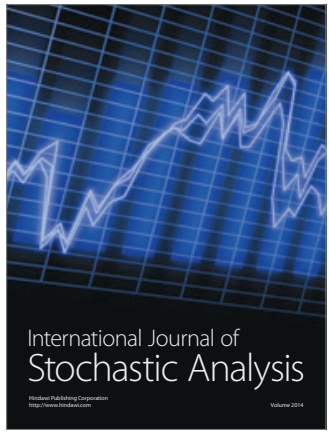

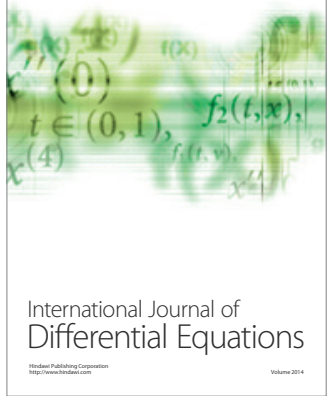
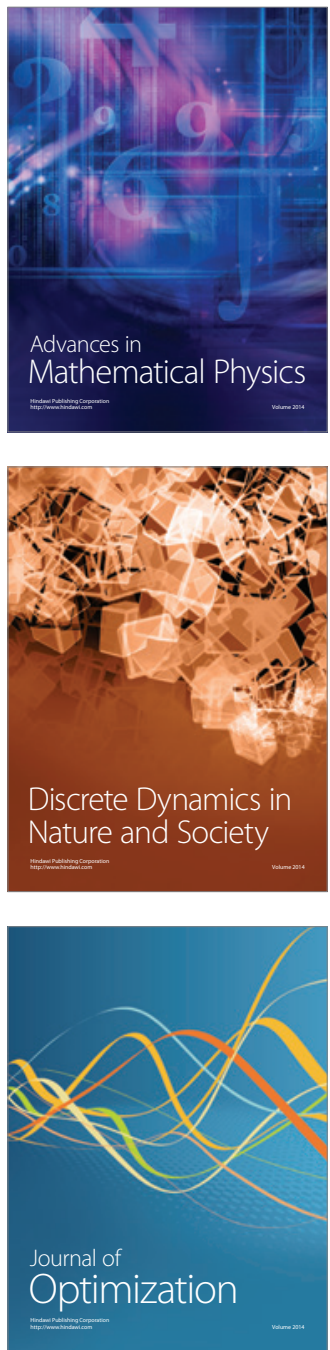